\title{
FERTILIZACIÓN CON N, P, K Y S, Y CURVAS DE ABSORCIÓN DE NUTRIMENTOS EN ARROZ VAR. CFX 18 EN GUANACASTE
}

\author{
Eloy Molina ${ }^{1 / *}$, José Hernán Rodríguez, \\ Palabras clave: Arroz, fertilización, absorción, nitrógeno, fósforo, potasio. \\ Keywords: Rice, fertilization, uptake, nitrogen, phosphorus, potassium.
}

\section{RESUMEN}

Se realizó un experimento de fertilización con N, P, K y S en arroz inundado, var. CFX 18, en un Inceptisol eútrico en Guanacaste y se determinó la curva de absorción de macro y micronutrimentos durante el ciclo de cultivo. Los tratamientos fueron de $0,80,120$ y $160 \mathrm{~kg} \cdot \mathrm{ha}^{-1} \mathrm{de} \mathrm{N}$; 0 , 40 y 80 kg.ha- ${ }^{-1}$ de $\mathrm{P}_{2} \mathrm{O}_{5} ; 0$ y 50 kg.ha ${ }^{-1}$ de $\mathrm{K}_{2} \mathrm{O}$, y 0 y $20 \mathrm{~kg} \cdot \mathrm{ha}^{-1}$ de $\mathrm{S}$, con un total de 8 tratamientos y 4 repeticiones. Se realizó un análisis foliar en prefloración y se determinó el rendimiento de arroz seco y limpio a la cosecha. En el tratamiento de 120 kg.ha-1 de N, 40 kg.ha ${ }^{-1}$ de $\mathrm{P}_{2} \mathrm{O}_{5}, 50$ kg.ha ${ }^{-1}$ de $\mathrm{K}_{2} \mathrm{O}$, y $20 \mathrm{~kg} \cdot \mathrm{ha}^{-1}$ de $\mathrm{S}$, se realizaron 5 muestreos de planta entera a los 28, 43, 66, 79 y 93 días de la germinación, para determinar peso seco, análisis químico de nutrimentos y curvas de absorción en función de la edad de la planta. Hubo respuesta significativa en rendimiento al N, P, K y S. El tratamiento de $120 \mathrm{~kg} \cdot \mathrm{ha}^{-1}$ de N, $40 \mathrm{~kg} \cdot \mathrm{ha}^{-1}$ de $\mathrm{P}_{2} \mathrm{O}_{5}, 50 \mathrm{~kg} \cdot \mathrm{ha}^{-1}$ de $\mathrm{K}_{2} \mathrm{O}$, y $20 \mathrm{~kg} \cdot \mathrm{ha}^{-1}$ de S, presentó el rendimiento más alto de arroz en granza, en un suelo que tenía un contenido adecuado de $\mathrm{P}$ y $\mathrm{K}$, pero bajo en $\mathrm{S}$. Estas mismas dosis propiciaron los contenidos de $\mathrm{N}$ y $\mathrm{P}$ foliar más altos, con diferencia significativa respecto a los tratamientos $\sin \mathrm{N}$ y sin P. La curva de absorción

\footnotetext{
1 Autor para correspondencia. Correo electrónico: eloy.molina@ucr.ac.cr

* Centro de Investigaciones Agronómicas, Universidad de Costa Rica, San José Costa Rica.
}

\begin{abstract}
$\mathrm{N}, \mathbf{P}, \mathrm{K}$, and $\mathrm{S}$ fertilization and nutrient absorption curves on rice var. CFX 18 in Guanacaste. An experiment of fertilization with $\mathrm{N}, \mathrm{P}, \mathrm{K}$ and $\mathrm{S}$ on rice (cv. CFX 18) was evaluated under flooded conditions in a Eutric Inceptisol in Guanacaste; also, the absorption curve of macro and micronutrients during the growing season was determined. The treatments were $0,80,120$ and $160 \mathrm{~kg} \cdot \mathrm{ha}^{-1}$ of $\mathrm{N} ; 0,40$ and $80 \mathrm{~kg} \cdot \mathrm{ha}^{-1}$ of $\mathrm{P}_{2} \mathrm{O}_{5}$; 0 and $50 \mathrm{~kg} \cdot \mathrm{ha}^{-1}$ of $\mathrm{K}_{2} \mathrm{O}$, and 0 and $20 \mathrm{~kg} \cdot \mathrm{ha}^{-1}$ of $\mathrm{S}$, for a total of 8 treatments with 4 replicates, in a randomized complete block design. Foliar analysis was performed at pre-flowering, and the yield of dry and clean grain was determined at harvest. In the treatment of $120 \mathrm{~kg} \cdot \mathrm{ha}^{-1}$ of $\mathrm{N}, 40$ kg.ha- $a^{-1}$ of $\mathrm{P}_{2} \mathrm{O}_{5}, 50 \mathrm{~kg} \cdot \mathrm{ha}^{-1}$ of $\mathrm{K}_{2} \mathrm{O}$, and $20 \mathrm{~kg} \cdot \mathrm{ha}^{-1}$ of S, 5 whole-plant samples were taken at 28 , 43, 66, 79 and 93 days after germination, for dry weight determination and chemical analysis of nutrients and for development of the absorption curves as a function of plant age. There was a significant response in yield to $\mathrm{N}, \mathrm{P}, \mathrm{K}$ and $\mathrm{S}$. The treatment of $120 \mathrm{~kg} \cdot \mathrm{ha}^{1}$ of N, $40 \mathrm{~kg} \cdot \mathrm{ha}^{-1}$ of $\mathrm{P}_{2} \mathrm{O}_{5}, 50 \mathrm{~kg} \cdot \mathrm{ha}^{-1}$ of $\mathrm{K}_{2} \mathrm{O}$, and $20 \mathrm{~kg} \cdot \mathrm{ha}^{-1}$ of $\mathrm{S}$ presented the highest grain yield, in a soil that had an adequate content of $\mathrm{P}$ and $\mathrm{K}$, but low in $\mathrm{S}$.
\end{abstract}

** Consultor privado, Liberia, Guanacaste, Costa Rica. 
de nutrimentos estuvo relacionada directamente con la producción de biomasa. La mayor parte de la absorción de nutrimentos se presentó en la etapa reproductiva y de maduración. La absorción máxima de N, P, K, Ca, Mg y S fue de 61, 25, 197, 17, 11 y 9 kg.ha- ${ }^{-1}$, respectivamente.

\section{INTRODUCCIÓN}

La actividad arrocera en Costa Rica es de gran importancia dentro del sector agrícola, donde la necesidad de lograr altos rendimientos sin un incremento desmedido en los costos de producción ha obligado a mantener activa la investigación en las prácticas culturales del cultivo, con la utilización de insumos y tecnologías modernas, donde la fertilización es uno de los rubros más importantes. Como país productor de arroz con más de 60000 ha anuales de producción, se hace imperante la necesidad de innovar con técnicas y variedades que permitan ser más eficientes para enfrentar las condiciones actuales del mercado y la competencia a nivel internacional (Villegas et al. 2008).

El arroz en Costa Rica se cultiva en una gran diversidad de suelos con características físico-químicas muy variables, que van desde los suelos aluviales ricos en Ca y $\mathrm{Mg}$ que predominan en el Sur, Pacífico Central y Guanacaste, hasta los suelos ácidos bajos en bases intercambiables de la Zona Norte (Murillo y González 1983). La fertilización del cultivo normalmente se maneja con la aplicación de N, P, K, S y Zn, en donde las fuentes y épocas dependen de los tipos de suelo y de las condiciones del clima (Cordero 1993).

Dentro del uso de insumos en el cultivo del arroz, la fertilización constituye un factor importante en la obtención de altos rendimientos. La respuesta a la fertilización depende de la variedad, la fertilidad del suelo, el clima, el manejo
These same treatments brought about the higher foliar contents of $\mathrm{N}$ and $\mathrm{P}$ and were significantly different from treatments with no $\mathrm{N}$ and no $\mathrm{P}$. The nutrient absorption curve was directly related to biomass production. Most of the absorption of nutrients occurred during the reproductive and ripening stages. The maximum absorption of $\mathrm{N}$, $\mathrm{P}, \mathrm{K}, \mathrm{Ca}, \mathrm{Mg}$ and $\mathrm{S}$ was $61,25,197,17,11$ and 9 $\mathrm{kg} \cdot \mathrm{ha}^{-1}$, respectively.

del agua, y el control de plagas y enfermedades (Cordero 1991).

El $\mathrm{N}$ es el elemento más importante en los programas de fertilización del arroz, ya que cumple un papel determinante en el desarrollo de la planta, debido a que forma parte de la estructura molecular de las proteínas, de las lecitinas, de la clorofila, de los ácidos nucleicos (ADN y ARN) de los citocromos y de las coenzimas (Dobermann y Fairhurst 2000). El N promueve el crecimiento vegetativo, el macollamiento, la formación y llenado del grano, y mejora el rendimiento (De Datta 1981). Su deficiencia se manifiesta con plantas pequeñas y atrofiadas, hojas pequeñas, angostas y erectas, clorosis o amarillamiento del tejido foliar que inicia en las hojas más viejas, reducción del macollamiento, y disminución del rendimiento de grano (Yoshida 1981, Fageria et al. 1995). Las recomendaciones de fertilización nitrogenada dependen del sistema de cultivo empleado y la variedad. Normalmente el arroz de secano requiere dosis bajas de $\mathrm{N}$ que oscilan entre $70-120 \mathrm{~kg} \cdot \mathrm{ha}^{-1}$ de $\mathrm{N}$, en tanto que en arroz bajo riego la dosis sube a un rango de 120-160 kg.ha-1 de N (Cordero 1991, Coto 1991). Las recomendaciones de $\mathrm{N}$ para arroz bajo riego en Arkansas oscilan entre 100 y 200 kg.ha ${ }^{-1}$, y dependen del ciclo de la variedad (Wilson et al. 2010). El fraccionamiento de la fertilización nitrogenada es una de las alternativas que sirven para mejorar la eficiencia de fertilizante. La aplicación de $\mathrm{N}$ debe distribuirse en las etapas de mayor demanda del nutriente por parte de la 
planta de arroz, las cuales corresponden a inicio de macollamiento, inicio de formación del primordio floral o prefloración, y en floración (Dobermann y Fairhurst 2000). Para una variedad de 125 días de duración, el fraccionamiento del $\mathrm{N}$ se podría distribuir a 25-35 días, 45-55 días, 65-75 días después de la siembra, respectivamente (Cordero 1993).

El P es muy importante para el desarrollo radicular, crecimiento vegetativo, floración y desarrollo del grano, pues además es componente de los ácidos nucleicos, fosfolípidos, así como de las membranas celulares (Yoshida 1981). Cumple una función importante en el metabolismo energético, debido a que es parte constituyente de la molécula de ATP (adenosín trifosfato) (Fageria et al. 1995). También es parte integral de las coenzimas NAD (nicotinamida adenín dinucleótido) y NADP (nicotinamida adenín dinucleótido fosfato), que cumplen una función fundamental en la fotosíntesis, glucólisis, respiración y síntesis de ácidos grasos (Dobermann y Fairhurst 2000). La deficiencia de $\mathrm{P}$ se manifiesta como la aparición al inicio de un color verde oscuro en hojas viejas, que se tornan luego de color amarillo-anaranjado. Las plantas lucen atrofiadas, con un limitado macollamiento, y se reduce la longitud de las hojas y el número de panojas (Rodríguez 1999). Las recomendaciones de $\mathrm{P}$ en arroz en Costa Rica oscilan en un rango de 30-60 kg.ha-1 de $\mathrm{P}_{2} \mathrm{O}_{5}$, y generalmente se aplica a la siembra, para aprovechar la incorporación del fertilizante en banda con el uso de la abonadora (Cordero 1993). Los suelos con contenidos inferiores a $10 \mathrm{mg} . \mathrm{l}^{-1} \mathrm{de}$ $\mathrm{P}$ disponible responden a las dosis más altas de $\mathrm{P}$, en tanto que un valor superior a $15 \mathrm{mg} \cdot \mathrm{l}^{-1} \mathrm{de}$ $\mathrm{P}$ se considera óptimo para el cultivo (Cordero y Miner 1975).

La función principal del $\mathrm{K}$ en el cultivo de arroz es la regulación hídrica de la planta y el aumento de la resistencia a plagas y enfermedades como Pyricularia y Heminthosporium (De Datta 1981). En forma general, el K está relacionado con procesos muy importantes como la fotosíntesis, respiración, formación de clorofila, metabolismo de carbohidratos y activador de enzimas necesarias en la síntesis de proteínas (Dobermann y Fairhurst 2000). El K también neutraliza los ácidos orgánicos y permite que la planta resista mejor las bajas temperaturas (Yoshida 1981). Muchos de los suelos aluviales altos en $\mathrm{Ca}$ y $\mathrm{Mg}$ presentan deficiencia de $\mathrm{K}$, principalmente por desbalance con $\mathrm{Ca}$ y $\mathrm{Mg}$ (Bertsch 1995). En algunos suelos como los Vertisoles, la deficiencia de $\mathrm{K}$ es más severa debido a la alta capacidad de fijación de $\mathrm{K}$ que poseen estos suelos con arcillas 2:1 de retículo expandible como la montmorillonita (Cordero 1993). Los síntomas de deficiencia de $\mathrm{K}$ causan que las plantas se atrofien, disminuye la capacidad de formación de hijos, y en las hojas más viejas se presentan una clorosis en los bordes y puntas que luego se transforma en necrosis. Los tallos son cortos y delgados, de menor peso y número de granos (Rodríguez 1999, Dobermann y Fairhurst 2000).

La dosis de $\mathrm{K}$ en los programas de fertilización es muy variable, ya que depende del resultado del análisis de suelo y varía entre 20 y $75 \mathrm{~kg}$ ha $^{-1}$ de $_{2} \mathrm{O}$ (Cordero 1993), fraccionada en 2 aplicaciones al inicio del macollamiento y en la formación del primordio floral (Rodríguez 1999).

El azufre es de gran importancia para mejorar el aprovechamiento del nitrógeno por la planta (efecto sinergístico). Forma parte estructural de las proteínas y constituye los aminoácidos cistina, cisteína y metionina. Además forma parte de algunas vitaminas como la tiamina y biotina, de la coenzima A y de varias enzimas (Dobermann y Fairhurst 2000). La deficiencia de $\mathrm{S}$ es muy similar a la de $\mathrm{N}$, lo cual hace difícil su identificación visualmente. Un factor que la diferencia es que los síntomas se presentan en hojas más nuevas, al inicio las vainas foliares se tornan amarillas, avanza hacia las láminas de las hojas y provoca un amarillamiento general de la planta durante la etapa de formación de hijos. (Cordero 1993, Rodríguez 1999). Las recomendaciones de $S$ en arroz varían entre 15 y $30 \mathrm{~kg} \cdot \mathrm{ha}^{-1}$, y es frecuente encontrar problemas de $\mathrm{S}$ en suelos de origen aluvial (Cordero et al. 1986).

La variedad CFX 18 fue desarrollada en la Universidad de Lousiana y presenta resistencia 
a algunos herbicidas port-emergentes utilizados para el control de arroz rojo, una de las malezas más serias de los campos arroceros del país. Esta variedad no es tan productiva pero si es muy apropiada para sembrarla en tierras contaminadas con arroz rojo, por medio del sistema de producción de "Clearfield", que tiene como objetivo la limpieza de campos contaminados con semilla de esa maleza. CFX 18 es una variedad de ciclo corto (95-100 días), que hace necesario una programación más temprana de la fertilización en comparación con otras variedades tradicionales.

El objetivo de este trabajo fue evaluar la respuesta de la variedad CFX 18 a la fertilización con $\mathrm{N}, \mathrm{P}, \mathrm{K}, \mathrm{y} \mathrm{S}$, y determinar la curva de absorción de nutrimentos, en las condiciones de Guanacaste.

\section{MATERIALES Y MÉTODOS}

El experimento se realizó en la Finca El Pelón de la Bajura ubicada en El Salto, Liberia, Guanacaste. Esta zona tiene una elevación de 40 msnm, con una precipitación promedio de 1740 mm anuales, con temperaturas mínimas y máximas que oscilan desde los 23 a los $36^{\circ} \mathrm{C}$, para un promedio anual de $28^{\circ} \mathrm{C}$. El suelo es un Inceptisol de origen aluvial, de textura franca, buen drenaje, topografía plana, de fertilidad buena, con contenidos adecuados de $\mathrm{Ca}, \mathrm{Mg}$ y $\mathrm{K}$, y altos de $\mathrm{P}, \mathrm{y}$ niveles bajos de materia orgánica (Cuadro 1). Se utilizó la modalidad de riego con anegamiento en bancales independientes durante casi todo el ciclo de cultivo.

El ensayo consistió en la aplicación de diferentes combinaciones de N, P, K y S. El elemento principal en los ensayos fue el $\mathrm{N}$ dado que está bien fundamentado que este nutrimento es el más importante en la fertilización del cultivo. Se establecieron 4 dosis de N $(0,80,120$ y 160 kg.ha ${ }^{-1}$ de $\left.\mathrm{N}\right), 3$ dosis de $\mathrm{P}\left(0,40\right.$ y $80 \mathrm{~kg}^{\circ} \mathrm{ha}^{-1}$ de $\left.\mathrm{P}_{2} \mathrm{O}_{5}\right), 2$ de $\mathrm{K}\left(0\right.$ y $50 \mathrm{~kg} \cdot \mathrm{ha}^{-1}$ de $\left.\mathrm{K}_{2} \mathrm{O}\right)$, y 2 de $\mathrm{S}$ (0 y $\left.20 \mathrm{~kg} \cdot \mathrm{ha}^{-1}\right)$, como se aprecia en el Cuadro 2. La fertilización base cuando el elemento no fue variable o tratamiento fue de $120 \mathrm{~kg} \cdot \mathrm{ha}^{-1} \mathrm{de} \mathrm{N}, 40$ kg.ha- ${ }^{-1}$ de $\mathrm{P}_{2} \mathrm{O}_{5}, 50 \mathrm{~kg} \cdot \mathrm{ha}^{-1}$ de $\mathrm{K}_{2} \mathrm{O}$ y $20 \mathrm{~kg} \cdot \mathrm{ha}^{-1}$ de $\mathrm{S}$. Como fuentes de nutrimentos se utilizó urea, sulfato de amonio, Triple superfosfato, DAP, $\mathrm{KCl}$ y Sulfato de potasio.

Cuadro 1. Resultados de análisis de suelos, Finca El Pelón de la Bajura, Liberia, Guanacaste.

\begin{tabular}{|c|c|c|c|c|c|c|c|c|c|c|c|c|c|}
\hline \multirow[t]{2}{*}{ Ubicación } & \multirow{2}{*}{$\frac{\mathrm{pH}}{\mathrm{H}_{2} \mathrm{O}}$} & \multicolumn{5}{|c|}{$\operatorname{cmol}(+) \cdot 1^{-1}$} & \multirow{2}{*}{$\frac{\%}{\mathrm{SA}}$} & \multicolumn{6}{|c|}{$\operatorname{mg} .1^{-1}$} \\
\hline & & ACIDEZ & $\mathrm{Ca}$ & $\mathrm{Mg}$ & K & CICE & & $\mathrm{P}$ & S & $\mathrm{Zn}$ & $\mathrm{Cu}$ & $\mathrm{Fe}$ & $\mathrm{Mn}$ \\
\hline Finca El Pelón & 6,1 & 0,20 & 8,60 & 1,29 & 0,47 & 10,6 & 2 & 18 & 8 & 5,7 & 12 & 434 & 209 \\
\hline
\end{tabular}

Cuadro 2. Tratamientos del ensayo.

\begin{tabular}{|c|c|c|c|c|}
\hline Tratamiento & $\mathrm{N}$ & $\begin{array}{c}\mathrm{P}_{2} \mathrm{O}_{5} \\
\mathrm{~kg} \cdot \mathrm{ha}^{-1}\end{array}$ & $\mathrm{~K}_{2} \mathrm{O}$ & $S$ \\
\hline 1 & 0 & 40 & 50 & 20 \\
\hline 2 & 80 & 40 & 50 & 20 \\
\hline 3 & 120 & 40 & 50 & 20 \\
\hline 4 & 160 & 40 & 50 & 20 \\
\hline 5 & 120 & 0 & 50 & 20 \\
\hline 6 & 120 & 80 & 50 & 20 \\
\hline 7 & 120 & 40 & 0 & 20 \\
\hline 8 & 120 & 40 & 50 & 0 \\
\hline
\end{tabular}


Los nutrimentos se fraccionaron de la siguiente forma:

- $\quad$ El P se aplicó el $100 \%$ a la siembra.

- $\quad$ El N se fraccionó en 4 aplicaciones.

Siembra: $15 \%$.

15 días después de germinación (ddg): 25\%.

30 días ddg: $25 \%$.

45 días ddg: $35 \%$.

El K se fraccionó en 2 aplicaciones, $40 \%$ a los 15 días ddg y $60 \%$ a los 30 días ddg. El S se fraccionó en 2 aplicaciones en partes iguales a los 15 y 30 días ddg.

El fertilizante se aplicó al voleo sobre la superficie del suelo.

Los ensayos se establecieron con 8 tratamientos (Cuadro 2) y 4 repeticiones, con un diseño experimental de Bloques Completos al Azar. Cada parcela o unidad experimental tenía un tamaño $50 \mathrm{~m}^{2}$. La semilla se sembró al voleo con una voleadora, a 3,5 sacos.ha- $\mathrm{s}^{-1}$. Las prácticas de cultivo como preparación de suelos, control de malezas, manejo fitosanitario, etc, fueron las mismas que utilizó comercialmente la empresa Hacienda El Pelón de la Bajura.

Se realizó un muestreo foliar durante la etapa de pre-floración, a los 45 días ddg. Se tomó la segunda hoja de arriba hacia abajo, para el análisis químico de $\mathrm{N}, \mathrm{P}, \mathrm{Ca}, \mathrm{Mg}, \mathrm{K}, \mathrm{S}, \mathrm{Fe}, \mathrm{Cu}$, Zn, Mn y B, que se realizó en el Laboratorio de Suelos del CIA-UCR.

Se realizaron muestreos de planta entera para establecer las curvas de absorción de nutrimentos de la variedad. Los muestreos se realizaron en el tratamiento 3 , considerado de manera preliminar como un tratamiento óptimo de fertilización.

Los muestreos se tomaron en 5 épocas diferentes del crecimiento de la planta, que se detallan en el Cuadro 3.

Para hacer el muestreo se diseñó un marco de pvc de 0,5 m x 0,5 m, el cual se colocó aleatoriamente sobre el suelo en cada repetición del tratamiento 3 en las diferentes épocas de muestreo y se recogió todo el material vegetativo del arroz que se ubicaba dentro del marco. Posteriormente el material se separó en raíz, follaje, y granos, y se determinó el peso fresco y peso seco, y la concentración de nutrimentos en una sub-muestra molida. Con los datos de peso seco y contenido del nutrimento, se calculó la absorción de los elementos por hectárea y se diseñaron las curvas de absorción en función del tiempo de muestreo.

La cosecha de los ensayos se realizó en forma manual, al cortar con machete las espigas de arroz en el área útil de cada parcela experimental. Se cosechó en un área útil de $12 \mathrm{~m}^{2}$. El grano se separó de la espiga con una trilladora mecánica, se pesó y luego se tomó una sub-muestra para determinar el \% de humedad y calcular los valores de peso del arroz en granza seco y limpio.

Se realizó el análisis de varianza de la variable rendimiento de arroz en granza seco y limpio, con la aplicación del programa estadístico SAS (SAS 2009). Adicionalmente se hizo el análisis de significancia estadística con la prueba "t" y contrastes ortogonales. Los resultados del análisis foliar también fueron procesados de la misma forma.

Cuadro 3. Etapas fenológicas del cultivo del arroz, variedad CFX 18.

\begin{tabular}{lc}
\hline Etapa fenológica & Rango de tiempo (ddg) \\
\hline Macollamiento activo & 28 \\
Inicio primordio floral & 43 \\
Floración & 66 \\
Llenado de grano (lechoso) & 79 \\
Maduración & 93 \\
\hline
\end{tabular}




\section{RESULTADOS Y DISCUSIÓN}

\section{Rendimiento de grano}

En el Cuadro 4 se presentan los resultados de rendimiento de arroz en granza seco y limpio en kg.ha- ${ }^{-1}$. Los rendimientos estuvieron un poco por debajo de lo esperado debido a que no hubo un macollamiento óptimo desde el inicio, esto por cuanto el suministro de agua fue insuficiente en el área experimental durante algunos días por problemas de la finca que estuvieron fuera del control de los autores. Finalmente el suministro de agua se normalizó pero siempre afectó un poco el macollamiento.

Cuadro 4. Efecto de la fertilización con N, P, K y S en el rendimiento de arroz en granza de la variedad CFX 18 en Liberia, Guanacaste.

\begin{tabular}{cc}
\hline Tratamiento & $\begin{array}{c}\text { Rendimiento } \\
\mathrm{kg}^{-} \mathrm{ha}^{-1}\end{array}$ \\
\hline $3.120-40-50-20$ & $3923 \mathrm{a}$ \\
4. $160-40-50-20$ & $3702 \mathrm{ab}$ \\
$6.120-80-50-20$ & $3494 \mathrm{bc}$ \\
7. $120-40-0-20$ & $3367 \mathrm{~cd}$ \\
2. $80-40-50-20$ & $3123 \mathrm{de}$ \\
$5.120-0-50-20$ & $3013 \mathrm{e}$ \\
$8.120-40-50-0$ & $2873 \mathrm{e}$ \\
$1.0-40-50-20$ & $1613 \mathrm{~g}$ \\
\hline
\end{tabular}

Valores con las misma letra no difieren significativamente entre sí con $\mathrm{p}<0,001$ (Diferencia mínima significativa=333 $\left.\mathrm{kg} \cdot \mathrm{ha}^{-1}\right)$.

El análisis de varianza mostró que hubo diferencias significativas entre tratamientos $(\mathrm{p}<0,001)$. La prueba de diferencia mínima significativa (dms) permitió separar los tratamientos en orden de mayor a menor rendimiento según los resultados obtenidos.

La respuesta al $\mathrm{N}$ fue muy evidente y el tratamiento sin este elemento presentó el rendimiento más bajo, y su valor fue significativamente diferente del resto de los tratamientos. La dosis de $120 \mathrm{~kg}$.ha ${ }^{-1}$ de $\mathrm{N}$ presentó el rendimiento más alto, que superó en forma significativa a los tratamientos de 80 y $0 \mathrm{~kg} \cdot \mathrm{ha}^{-1}$ de $\mathrm{N}$, y muestran la importancia que tiene este elemento en la producción de arroz (Buzetti et al. 2006). Los resultados coinciden con las recomendaciones de Cordero (1993), quién menciona que el rango de respuesta del $\mathrm{N}$ oscila entre 80 y $180 \mathrm{~kg} \cdot \mathrm{ha}^{-1}$, según condiciones climáticas, edáficas y varietales.

La dosis de $160 \mathrm{~kg} \cdot \mathrm{ha}^{-1}$ de $\mathrm{N}$ presentó un rendimiento inferior a $120 \mathrm{~kg} \cdot \mathrm{ha}^{-1} \mathrm{de} \mathrm{N}$, aunque esa diferencia no fue estadísticamente significativa. Cordero (1991) encontró la máxima respuesta económica a la aplicación de $180 \mathrm{~kg} \cdot \mathrm{ha}^{-1}$ de $\mathrm{N}$ con la variedad CR 1821 en condiciones de riego, si bien esta variedad tiene un potencial de producción superior a CFX 18 y presenta una mayor capacidad de absorción de N (Cordero y Murillo 1990).

Los resultados de este estudio concuerdan con los datos presentados por Quirós y Ramírez (2006), quienes encontraron el mejor rendimiento de arroz en la variedad CR 1113 con una dosis de $120 \mathrm{~kg} \cdot \mathrm{ha}^{-1}$ de N, fraccionado en 3 aplicaciones durante el ciclo de crecimiento. Vargas (2002) encontró la máxima respuesta en rendimiento de arroz variedad Fedearroz 50, con una dosis de $180 \mathrm{~kg} \cdot \mathrm{ha}^{-1}$ en un Inceptisol alto en bases intercambiables de Parrita, bajo condiciones de secano.

La respuesta al $\mathrm{N}$ se observa con mayor claridad en la Figura 1, donde se presentan los resultados de los tratamientos de 0 a 160 kg.ha-1 de N, con la misma dosis de P, K y S.

Se hizo una medición de la eficiencia de fertilización para el mejor tratamiento de 120 kg.ha-1 de N:

Eficiencia de fertilización=(Rend. 0 N-Rend. 120 kg.ha- ${ }^{-1}$ de $\mathrm{N}$ )/dosis de $\mathrm{N}$

Eficiencia de fertilización $=(3923 \mathrm{~kg}-1613 \mathrm{~kg}) / 120 \mathrm{~kg}$ Eficiencia de fertilización=19,25 kg arroz. $\mathrm{kg}^{-1}$. ha $^{-1}$ de N

A pesar de que CFX 18 no es una variedad de alta producción, la eficiencia de fertilización fue de 19,25 $\mathrm{kg}$ de grano por cada $\mathrm{kg}$ de $\mathrm{N}$ agregado, que se considera buena. La eficiencia fue mayor que los datos reportados por Cordero (1991) quién encontró valores de eficiencia entre 


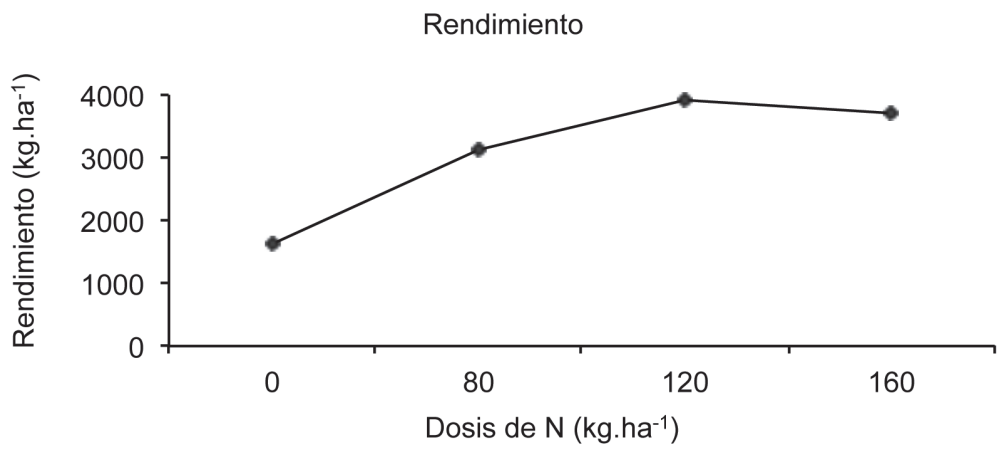

Fig. 1. Efecto de la dosis de $\mathrm{N}$ en el rendimiento del arroz variedad CFX18, Liberia, Guanacaste.

9 y $14 \mathrm{~kg}$ arroz. $\mathrm{kg}^{-1} \cdot \mathrm{ha}^{-1} \mathrm{de} \mathrm{N}$, en un experimento de fertilización nitrogenada con la variedad CR 1821.

Para el caso del P, los niveles de 40 y 80 kg.ha- ${ }^{-1}$ de $\mathrm{P}_{2} \mathrm{O}_{5}$ fueron significativamente superiores al tratamiento sin $\mathrm{P}$ (Cuadro 4). La respuesta a la fertilización con $\mathrm{P}$ se presentó a pesar de que el suelo tenía un nivel adecuado del elemento (18 mg. $\left.\mathrm{l}^{-1}\right)$, donde la dosis de $40 \mathrm{~kg} \cdot \mathrm{ha}^{-1}$ de $\mathrm{P}_{2} \mathrm{O}_{5}$ fue la de mejor respuesta agronómica. Los resultados no coinciden con las recomendaciones de Cordero (1993), quién indica que por encima de $10 \mathrm{mg} . \mathrm{l}^{-1}$ no se esperaría respuesta a la aplicación del elemento.

Vargas (2002) evaluó el efecto de diferentes dosis de $\mathrm{P}\left(0\right.$ a $90 \mathrm{~kg} \cdot \mathrm{ha}^{-1}$ de $\left.\mathrm{P}_{2} \mathrm{O}_{5}\right)$ en arroz variedad Fedearroz 50 en Parrita, y no encontró respuesta en rendimiento, atribuyéndolo al buen nivel inicial de $\mathrm{P}$ encontrado en el suelo (22 mg. $\left.1^{-1}\right)$.

Hubo respuesta a la fertilización con $\mathrm{K}$, debido a que las dosis de 0 y 50 kg.ha ${ }^{-1}$ de $\mathrm{K}_{2} \mathrm{O}$ (tratamientos 3 y 7), con las mismas dosis de $\mathrm{N}$, $\mathrm{P}$ y $\mathrm{S}$, mostraron diferencias significativas entre sí (Cuadro 4), a pesar de que el suelo presentó un contenido adecuado de $\mathrm{K}\left(0,47 \mathrm{cmol}(+) \cdot \mathrm{l}^{-1}\right)$. Vargas (2002) no encontró respuesta del arroz a la fertilización con $\mathrm{K}$ en un suelo de Parrita con 0,37 $\mathrm{cmol}(+) \cdot \mathrm{l}^{-1}$ Cordero (1993) recomienda la aplicación de $\mathrm{K}$ sólo en caso de suelos con 0,2 $\operatorname{cmol}(+) \cdot 1^{-1}$ o menos del elemento.
Los resultados muestran que hubo respuesta al S, al comparar los tratamientos 3 y 8 , en los que hubo diferencias significativas. Se destaca el hecho de que el tratamiento sin S presentó uno de los rendimientos más bajos del ensayo, sólo superó al tratamiento sin N. En este caso el resultado coincide con el nivel bajo de $\mathrm{S}$ en el suelo $\left(8 \mathrm{mg} . \mathrm{l}^{-1}\right)$, considerado un valor de deficiencia y que según Cordero (1993) requeriría una dosis de $15 \mathrm{~kg} \cdot \mathrm{ha}^{-1}$ de S. El efecto del S en arroz ha sido también documentado por Cordero et al. (1986), quienes encontraron respuesta al $\mathrm{S}$ en un suelo aluvial de Jacó, Puntarenas.

\section{Análisis foliar}

En el Cuadro 5 se presentan los resultados del análisis foliar realizado durante la fase final de macollamiento. En general no se presentaron contenidos deficientes de nutrimentos con excepción de $\mathrm{N}$ y $\mathrm{S}$ en algunos tratamientos.

El análisis de varianza mostró que hubo diferencias significativas entre tratamientos para los contenidos foliares de N, P, Mn y S. Para el caso del $\mathrm{N}$, la mayoría de los tratamientos presentaron valores por encima de $2,5 \%$, considerado el nivel mínimo de suficiencia para esa etapa de crecimiento del arroz (Dobermann y Fairhurst 2000). Los tratamientos con dosis de 120-160 kg.ha-1 de $\mathrm{N}$ mostraron el contenido más alto de $\mathrm{N}$, tales como el 3, 4, 5, 6 y 7, y de acuerdo con la prueba de diferencia mínima significativa no hubo diferencias estadísticas entre ellos (Cuadro 6). El tratamiento 
Cuadro 5. Resultado del análisis foliar realizado durante la fase de macollamiento en arroz var. CFX 18, Liberia, Guanacaste.

\begin{tabular}{|c|c|c|c|c|c|c|c|c|c|c|c|}
\hline \multirow{2}{*}{ Tratamiento } & \multicolumn{6}{|c|}{$(\%)$} & \multicolumn{5}{|c|}{$\mathrm{mg} \cdot \mathrm{kg}^{-1}$} \\
\hline & $\mathrm{N}$ & $\mathrm{P}$ & $\mathrm{Ca}$ & $\mathrm{Mg}$ & K & S & $\mathrm{Fe}$ & $\mathrm{Cu}$ & $\mathrm{Zn}$ & $\mathrm{Mn}$ & B \\
\hline 1. $0-40-50-20$ & 1,58 & 0,16 & 0,31 & 0,12 & 1,97 & 0,09 & 82 & 9 & 28 & 443 & 12 \\
\hline 2. $80-40-50-20$ & 2,39 & 0,18 & 0,29 & 0,12 & 2,06 & 0,18 & 96 & 11 & 36 & 527 & 9,8 \\
\hline 3. $120-40-50-20$ & 3,03 & 0,23 & 0,23 & 0,13 & 2,09 & 0,14 & 118 & 14 & 32 & 937 & 12,2 \\
\hline 4. $160-40-50-20$ & 2,78 & 0,19 & 0,25 & 0,12 & 1,88 & 0,16 & 105 & 12 & 26 & 436 & 16,3 \\
\hline 5. $120-0-50-20$ & 2,84 & 0,17 & 0,22 & 0,12 & 2,08 & 0,15 & 104 & 12 & 31 & 421 & 18,2 \\
\hline 6. $120-80-50-20$ & 2,68 & 0,21 & 0,36 & 0,12 & 1,93 & 0,2 & 103 & 13 & 27 & 630 & 9,5 \\
\hline 7. $120-40-0-20$ & 2,81 & 0,22 & 0,25 & 0,13 & 2,00 & 0,28 & 111 & 12 & 29 & 680 & 17,2 \\
\hline 8. $120-40-50-0$ & 2,49 & 0,16 & 0,58 & 0,14 & 2,13 & 0,11 & 87 & 61 & 55 & 141 & 14,5 \\
\hline
\end{tabular}

Cuadro 6. Efecto de la fertilización con N, P, K y S en el contenido foliar de N, P, S y Mn en hojas de arroz, Liberia, Guanacaste.

\begin{tabular}{llll}
\hline Tratamiento & $\% \mathrm{~N}$ & Tratamiento & $\mathrm{Mn}_{\left(\mathrm{mg} \cdot \mathrm{kg}^{-1}\right)}$ \\
\hline $3.120-40-50-20$ & $3,03 \mathrm{a}$ & $3.120-40-50-20$ & $937 \mathrm{a}$ \\
$5.120-0-50-20$ & $2,84 \mathrm{ab}$ & $7.120-40-0-20$ & $680 \mathrm{~b}$ \\
$7.120-40-0-20$ & $2,81 \mathrm{abc}$ & $6.120-80-50-20$ & $630 \mathrm{bc}$ \\
$4.160-40-50-20$ & $2,78 \mathrm{abcd}$ & $2.80-40-50-20$ & $527 \mathrm{bcd}$ \\
$6.120-80-50-20$ & $2,68 \mathrm{abcd}$ & $1.0-40-50-20$ & $443 \mathrm{~d}$ \\
$8.120-40-50-0$ & $2,49 \mathrm{bcd}$ & $4.160-40-50-20$ & $436 \mathrm{de}$ \\
$2.80-40-50-20$ & $2,39 \mathrm{~d}$ & $5.120-0-50-20$ & $421 \mathrm{e}$ \\
$1.0-40-50-20$ & $1,58 \mathrm{e}$ & $8.120-40-50-0$ & $141 \mathrm{f}$ \\
\hline Tratamiento & $\% \mathrm{P}$ & Tratamiento & $\% \mathrm{~S}$ \\
\hline $3.120-40-50-20$ & $0,23 \mathrm{a}$ & $7.120-40-0-20$ & $0,28 \mathrm{a}$ \\
$7.120-40-0-20$ & $0,22 \mathrm{ab}$ & $6.120-80-50-20$ & $0,20 \mathrm{~b}$ \\
$6.120-80-50-20$ & $0,21 \mathrm{bc}$ & $2.80-40-50-20$ & $0,18 \mathrm{bc}$ \\
$4.160-40-50-20$ & $0,19 \mathrm{~cd}$ & $4.160-40-50-20$ & $0,16 \mathrm{bcd}$ \\
$2.80-40-50-20$ & $0,18 \mathrm{de}$ & $5.120-0-50-20$ & $0,15 \mathrm{bcd}$ \\
$5.120-0-50-20$ & $0,17 \mathrm{ef}$ & $3.120-40-50-20$ & $0,14 \mathrm{~cd}$ \\
$1.0-40-50-20$ & $0,16 \mathrm{ef}$ & $8.120-40-50-0$ & $0,11 \mathrm{de}$ \\
$8.120-40-50-0$ & $0,16 \mathrm{ef}$ & $1.0-40-50-20$ & $0,09 \mathrm{e}$ \\
\hline
\end{tabular}

Tratamientos con la misma letra no difieren significativamente con $\mathrm{p}<0,001$.

sin $\mathrm{N}$ presentó la concentración más baja del elemento con un valor de $1,58 \%$ y fue inferior en forma significativa del resto de tratamientos, seguido a continuación por la dosis baja de $\mathrm{N}$ $\left(80 \mathrm{~kg} \cdot \mathrm{ha}^{-1}\right)$ (Cuadro 6). Cuando no se adicionó
$\mathrm{S}$, el nivel de $\mathrm{N}$ fue también muy bajo, como se observa en el resultado del tratamiento 8 , lo que probablemente se debe al efecto sinergístico del S que ayuda a mejorar la asimilación del $\mathrm{N}$ (Marschner 1995). 
El contenido de $\mathrm{P}$ foliar presentó valores por encima del nivel crítico para arroz. Sin embargo, su concentración fue afectada en forma significativa por los tratamientos de fertilización (Cuadro 6), que coincidió con la respuesta encontrada en rendimiento a la dosis de $40 \mathrm{~kg} \cdot \mathrm{ha}^{-1}$ de $\mathrm{P}_{2} \mathrm{O}_{5}$. Se destaca el hecho que 2 de los tratamientos que presentaron el nivel más bajo de $\mathrm{P}$ fueron la dosis 0 de $\mathrm{N}$ y $\mathrm{S}$, lo que indica la importancia que tienen estos 2 elementos en la asimilación de P por la planta.

El contenido de $\mathrm{S}$ foliar también presentó diferencias significativas (Cuadro 6), y la mayoría de los tratamientos presentaron un nivel inferior al nivel mínimo de $0,2 \%$. De acuerdo con la prueba de diferencia mínima significativa, las concentraciones más bajas de $\mathrm{S}$ se presentaron en los tratamientos sin N y S (Cuadro 6). Se destaca el tratamiento sin $\mathrm{K}$ que mostró el nivel más alto de $\mathrm{S}$ foliar, probablemente debido a un antagonismo entre ambos elementos. Con base en los contenidos bajos de $\mathrm{S}$ foliar, es probable que la dosis de $S$ utilizada fuera insuficiente, lo que se confirma por la respuesta encontrada.

Hubo diferencias significativas por efecto de tratamiento en la concentración de Mn foliar, las cuales probablemente se debieron más al efecto del manejo del agua que a los mismos tratamientos, ya que por coincidencia el tratamiento 3 en algunas parcelas se acumuló más el agua que en otras debido a diferencias en nivelación del terreno y por su ubicación.

\section{Curvas de absorción de nutrimentos}

La absorción de nutrimentos en el tiempo estuvo directamente relacionada con la producción de biomasa. Para el caso del N, la absorción del elemento se incrementó desde la germinación hasta alcanzar el valor máximo a los 79 días (Figura 2), con una absorción total de 61 kg.ha ${ }^{-1}$. La absorción de $\mathrm{N}$ estuvo limitada por el rendimiento ligeramente bajo obtenido en el tratamiento 3 (3,9 t.ha $\left.{ }^{-1}\right)$. La etapa de mayor absorción de $\mathrm{N}$ se ubicó entre los 28 y 79 días después de la germinación, período en el que se absorbió el 52\% del $\mathrm{N}$ total. La máxima absorción de $\mathrm{N}$ coincidió con la fase de llenado en la etapa de grano lechoso. La absorción de $\mathrm{N}$ disminuyó después de los 79 días, debido a la maduración y finalización del llenado de grano, y la pérdida de biomasa área. La variedad CFX 18 presentó una absorción de $\mathrm{N}$ muy temprana debido a su ciclo corto. El $48 \%$ del $\mathrm{N}$ fue absorbido en los primeros 28 días después de germinación y coincide con la etapa de macollamiento activo.

Un posible esquema de aplicación de $\mathrm{N}$ en CFX 18 sería: 15, 30, 45 y 55 días después de la germinación, pues en ese caso coincidirá con las etapas de inicio de macollamiento, macollamiento activo, inicio de primordio y pre-floración,

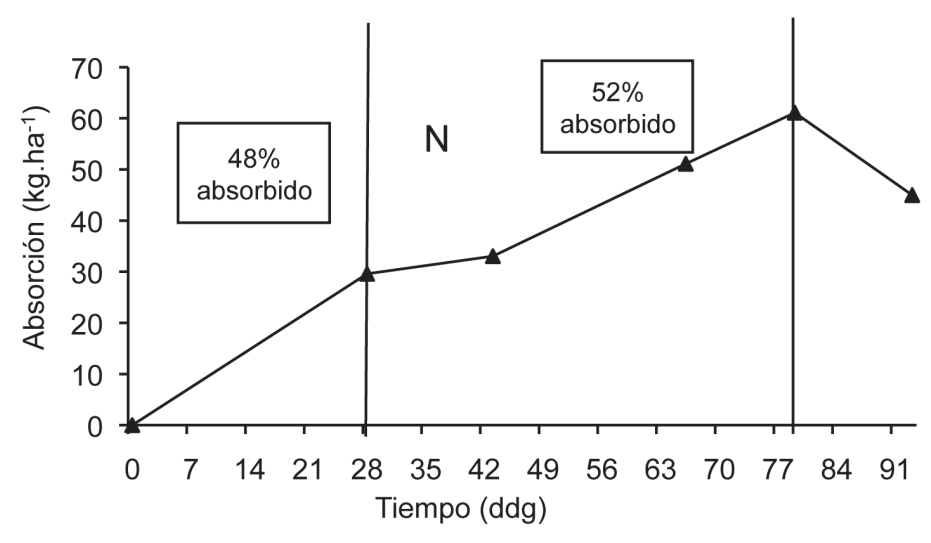

Fig. 2. Curva de absorción total de N en var. CFX 18, Liberia, Guanacaste. 
respectivamente, tal y como lo indican Dobermann y Fairhurst (2000) y Cordero (1993).

La absorción de $\mathrm{P}$ fue ascendente hasta los 66 días (Figura 3) en el que alcanza su valor máximo total de $12 \mathrm{~kg} \cdot \mathrm{ha}^{-1}$. Durante la etapa de crecimiento vegetativo (hasta el día 43) se absorbió el $42 \%$ de los requerimientos totales de P. A partir del inicio de la formación del primordio y hasta la floración, la planta absorbió el 58\% del P total requerido. Entre los 28 y 66 días la planta absorbió más del $80 \%$ de sus requerimientos de $\mathrm{P}$, demostró que la mayor parte de la absorción de $\mathrm{P}$ es tardía, y que se concentra durante la etapa reproductiva. Resultados similares han sido reportados por Molina (citado por Berstch 2003) en variedades como CR 1113, Camago 8, Anabela,
Llano 5, 4338 y 4102. En un estudio realizado por Vargas (2002), la absorción total de de P en la variedad Fedearroz 50 fue de $45 \mathrm{~kg}^{\mathrm{k}} \mathrm{ha}^{-1}$ y cerca del $60 \%$ se presentó durante la etapa reproductiva. La fertilización con P en arroz se realiza a la siembra o poco después de la siembra (Cordero 1991), principalmente para favorecer la colocación del fertilizante y disminuir las pérdidas por fijación, y para mejorar el anclaje de la planta desde el inicio del crecimiento (Vargas 2002). Sin embargo, las curvas de absorción indican que el $P$ podría ser aplicado también durante el macollamiento e inicio de la fase reproductiva.

La absorción de $\mathrm{K}$ fue ascendente durante casi todo el ciclo del cultivo hasta la maduración del grano (Figura 4), hasta alcanzar un valor

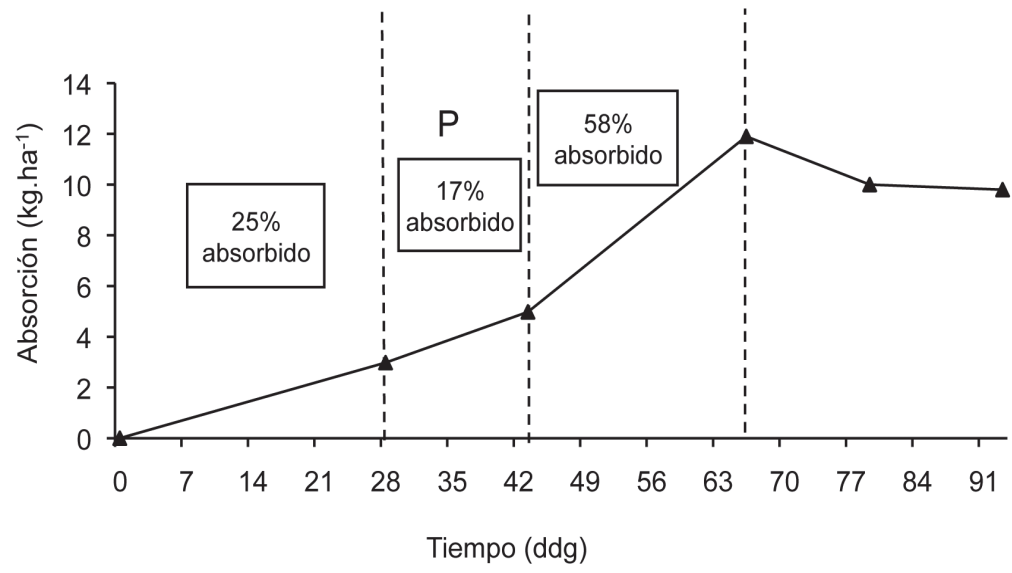

Fig. 3. Curva de absorción total de P en var. CFX 18, Liberia, Guanacaste.

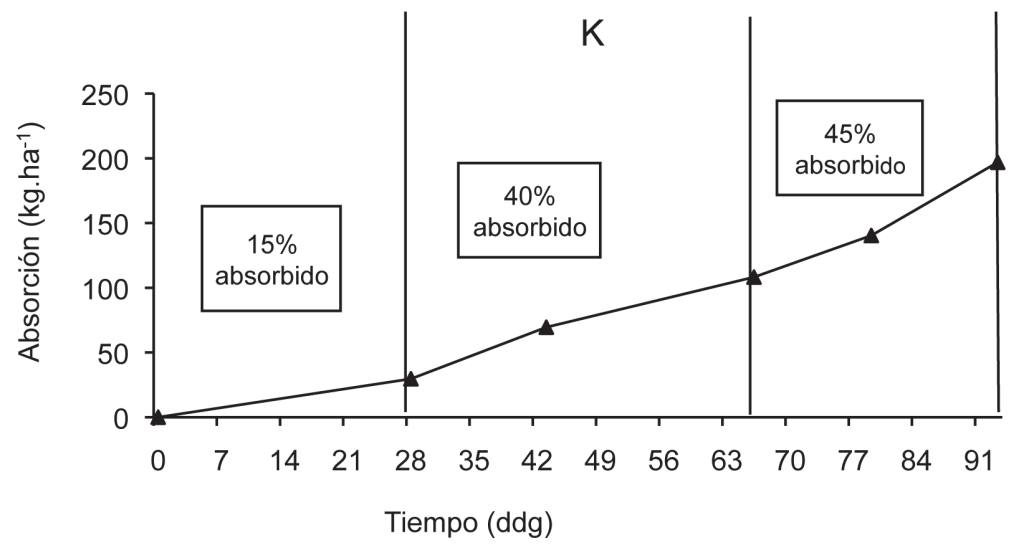

Fig. 4. Curva de absorción total de K en var. CFX 18, Liberia, Guanacaste. 
máximo de $197 \mathrm{~kg} \cdot \mathrm{ha}^{-1}$. Al inicio del crecimiento y hasta la etapa de macollamiento activo (28 ddg), la planta absorbió el $15 \%$ del $\mathrm{K}$ total requerido. Desde ese momento y hasta la floración (66 ddg), la planta absorbió el $40 \%$ del $\mathrm{K}$ total. Y finalmente se presentó una absorción tardía muy alta durante la fase de maduración y que correspondió al $45 \%$ del $\mathrm{K}$ total absorbido. Un resultado similar encontró Vargas (2002) en la variedad Fedearroz 50 , en la que más del $80 \%$ del $\mathrm{K}$ fue absorbido en la fase reproductiva y de maduración.

En la Figura 5 se presenta la absorción de $\mathrm{Ca}, \mathrm{Mg}$ y S. El patrón de absorción de Ca fue muy similar al de $\mathrm{N}$ y $\mathrm{P}$, y la mayor parte de la absorción de Ca ocurrió entre los 28 y 79 días. El valor máximo de absorción fue de $17 \mathrm{~kg} \cdot \mathrm{ha}^{-1}$. La extracción máxima de $\mathrm{Mg}$ fue de $11 \mathrm{~kg} \cdot \mathrm{ha}^{-1}$, valor similar a la absorción de P. Ambos nutrimentos mantuvieron una curva de absorción ascendente hasta llegar a la fase de maduración. El S también tuvo un comportamiento de absorción similar al $\mathrm{N}$, con un valor máximo absorbido de $9 \mathrm{~kg} \cdot \mathrm{ha}^{-1} \mathrm{a}$ los 79 días.

Para el caso de los micronutrimentos, el Fe y Mn fueron absorbidos en mayor cantidad que los otros, con valores 5,7 y $6,9 \mathrm{~kg} \cdot \mathrm{ha}^{-1}$, respectivamente, en su pico máximo de absorción a los 93 días (Figura 6), la mayor parte de esta absorción se presentó en la raíz debido a que la planta acumula ambos nutrimentos en este

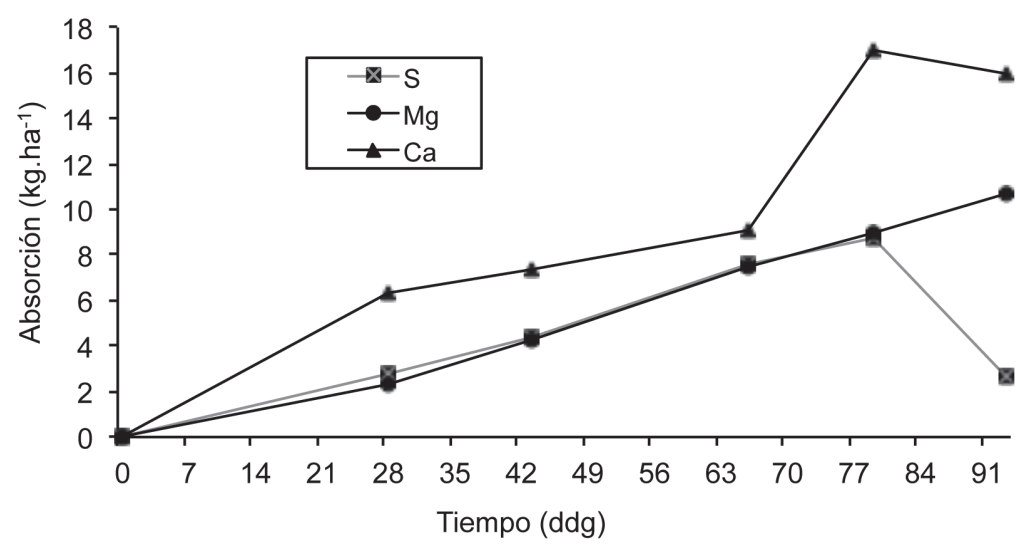

Fig. 5. Curvas de absorción total de Ca, Mg y S en var. CFX 18, Liberia Guanacaste.

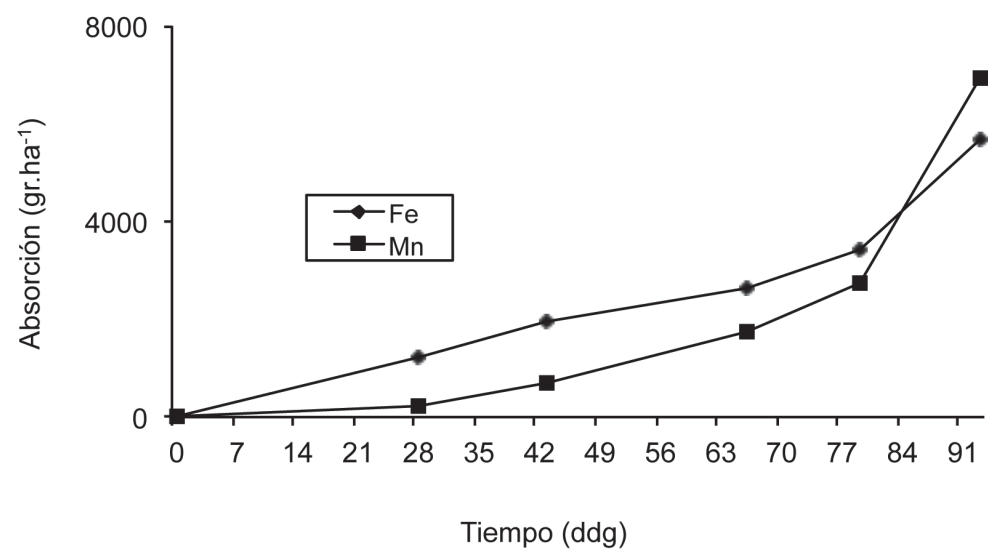

Fig. 6. Curva de absorción total de Fe y Mn en var. CFX 18, Liberia, Guanacaste. 
órgano (Dobermann y Fairhurst 2000). El mayor incremento en la absorción se presentó durante la etapa de llenado del grano.

La absorción de Cu, Zn y B se incrementó a partir de los 28 días y alcanzó los valores más altos durante la maduración (Figura 7). La mayor parte se absorbió durante la fase reproductiva y de maduración. La absorción máxima de $\mathrm{Cu}, \mathrm{Zn}$ y B fue de 345, 371 y 64 g.ha ${ }^{-1}$, respectivamente.
Para efectos de aplicación foliar de micronutrimentos como $\mathrm{Zn}$ y B, el mejor momento es al inicio del primordio, en pre-floración y en floración.

El orden de absorción de nutrimentos de la variedad CFX 18 bajo las condiciones de Guanacaste para una producción de biomasa fresca de 18 t.ha $^{-1}$ y un rendimiento promedio de 3,9 t.ha ${ }^{-1}$ de arroz seco y limpio fue de: $\mathrm{K}>\mathrm{N}>\mathrm{Ca}>\mathrm{P}>\mathrm{Mg}>\mathrm{S}>\mathrm{Mn}>\mathrm{Fe}>\mathrm{Zn}>\mathrm{Cu}>\mathrm{B}$.

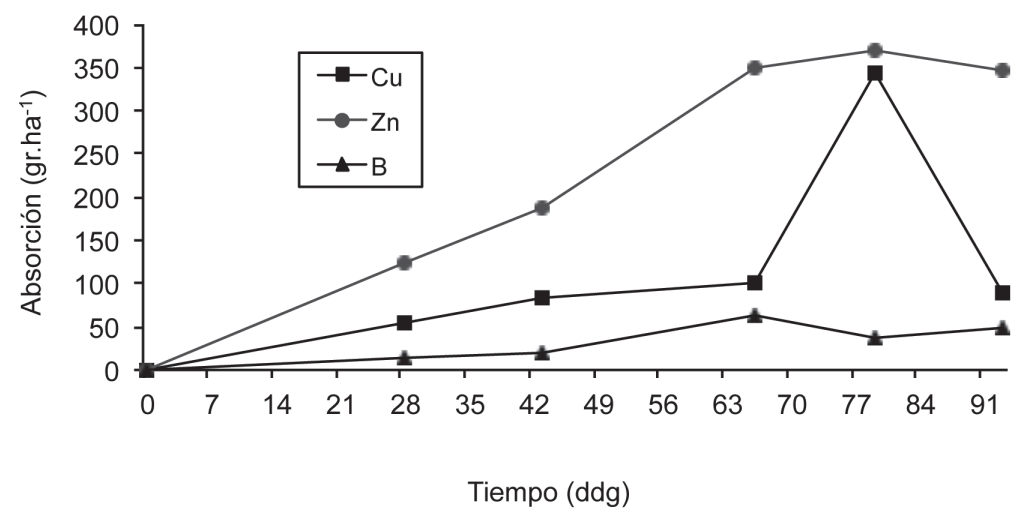

Fig. 7. Curva de absorción total de Cu, Zn y B en var. CFX 18, Liberia, Guanacaste.

\section{LITERATURA CITADA}

BERTSCH F. 1995. La fertilidad de los suelos y su manejo. Asociación Costarricense de la Ciencia del Suelo. San José, Costa Rica. 157 p.

BERTSCH F. 2003. Absorción de nutrimentos por los cultivos. ACCS, San José, Costa Rica. 307 p.

BUZETTI S., BAZANINI G., FREITAS J., ANDREOTTI M., ARF O., EUSTÁQUIO DE SÁ M., ANDRADE F. 2006. Resposta de cultivares de arroz a doses de nitrogênio e do regulador de crescimento cloreto de clormequat. Pesquisa Agropecuária Brasileira 41(12):1731-1737.

CORDERO A. 1991. La fertilización del cultivo de arroz bajo riego en Costa Rica, pp. 196-205. In: T.J. Smyth, W.R. Raun y F. Bertsch (eds), Segundo Taller Latinoamericano de Manejo de Suelos Tropicales, North Carolina State University. San José, Costa Rica.

CORDERO A. 1993. Fertilización y nutrición mineral del arroz. Editorial de la Universidad de Costa Rica, San José, Costa Rica. 100 p.
CORDERO A., MINER G.S. 1975. Programa de calibración de análisis de suelo para la fertilización de arroz y maíz en Costa Rica. En Seminario sobre manejo de suelos y el proceso de desarrollo en América Tropical. Manejo de Suelos en América Tropical. San José, Costa Rica. p. 533-548.

CORDERO A., MURILLO I. 1990. Remoción de nutrimentos por el cultivar de arroz CR-1821 bajo inundación. Agronomía Costarricense 14(1):79-84.

CORDERO A., MURILLO I., MOLINA E. 1986. Deficiencia de azufre: nuevo factor limitante en la producción de arroz de secano en la zona de Jacó, Garabito, Puntarenas. In $7^{\circ}$ Congreso Agronómico Nacional, $23^{\circ}$ Congreso de Horticultura ASHS Región Tropical, Resúmenes. Colegio de Ingenieros Agrónomos, San José, Costa Rica. p. 8-9.

COTO P. 1991. Respuesta de la variedad de arroz (Oryza sativa L) CR 1821 y las líneas promisorias CR 8334 y CR 8341, a la fertilización nitrogenada bajo condiciones de riego. Tesis Ingeniero Agrónomo, Universidad de Costa Rica, San José, Costa Rica. $103 \mathrm{p}$. 
DE DATTA S.K. 1981. Principles and Practices of Rice Productions. John Willey \& Son. New York. 618 p.

DOBERMANN A., FAIRHURST T. 2000. Rice: nutrient disorders and nutrient management. Potash and Phosphate Institute and Internacional Rice Research Institute, USA. 190 p.

FAGERIA N.K., FERREIRA E., PRABHU A.S., BARBOZA M.P., FILIPPI M.C. 1995. Seja o doutor do seu arroz. Arquivo do Agrônomo No. 10. POTAFOS, Brasil. $22 \mathrm{p}$.

MARSCHNER H. 1995. Mineral nutrition of higher plants. $2^{\circ}$ ed, Academic Press, New York. 674 p.

MURILlO J., GONZÁLEZ R. 1983. Manual para la producción de arroz de secano en Costa Rica. CAFESA. San José, Costa Rica. 127 p.

QUIRÓS R., RAMÍREZ C. 2005. Evaluación de la fertilización nitrogenada en arroz inundado. Agronomía Mesoamericana 17(2):179-188.

RODRÍGUEZ J.H. 1999. Fertilización del cultivo del arroz (Oryza sativa). In $11^{\circ}$ Congreso Nacional Agronómico y Recursos Naturales, $3^{\circ}$ Congreso Nacional de
Suelos. Colegio de Ingenieros Agrónomos, San José Costa Rica. Vol. III, p. 123-136.

SAS. 2009. SAS/STAT Software. Release 9.2. SAS Institute Inc.,Cary, North Carolina.

VARGAS M. 2002. Fertilización con cuatro niveles de nitrógeno, fósforo y potasio y curvas de absorción de la variedad Fedearroz 50 en condiciones de secano favorecido. CONARROZ, San José, Costa Rica. 24 p.

VILLEGAS O., VARGAS F., PÉREZ J., GARCÍ R., PORRAS S., MENESES R., QUESADA A., DELGADO G., ALPIZAR D., MORA B., LEON R., ALFARO D. 2008. Programa nacional de producción de alimentos región Huetar Norte. Boletín técnico, MAG, San José, Costa Rica. 35 p.

WILSON C.E., SLATON N.A., NORMAN R.J. 2010 Nitrogen fertilization of rice in Arkansas. Cooperative Extension Service Bulletin, University of Arkansas, USA. 8 p.

YOSHIDA S. 1981. Fundamental of Rice Crop Science. International Rice Research Institute. Los Baños, Filipinas. 267 p. 
